\title{
Ciliary defects and genetics of primary ciliary dyskinesia
}

Estelle Escudier ${ }^{1}, \mathrm{MD} \mathrm{PhD}$ : estelle.escudier@trs.aphp.fr

Philippe Duquesnoy ${ }^{1}$, BA: philippe.duquesnoy@trs.aphp.fr

Jean François Papon ${ }^{1,2}$, MD: jean-francois.papon@hmn.aphp.fr

Serge Amselem ${ }^{1}$, MD PhD: serge.amselem@trs.aphp.fr

\section{Address:}

1. AP-HP, Service de Génétique et d'Embryologie médicales and Inserm U.933

Hôpital Armand-Trousseau

26, avenue du Docteur Arnold-Netter

75571 Paris Cedex 13, France

Phone: + 33144735239

Fax: + 33144735219

2. AP-HP, Service d'ORL et de chirurgie cervico-faciale, Groupe hospitalier Henri-Mondor et Hôpital intercommunal and Inserm U.955 Créteil, F-94010, France

Phone: +33149812225

Fax: + 33149812423

\section{Acknowledgments:}

This work was supported by grants from the Legs Poix from the Chancellerie des Universités, the Assistance Publique-Hôpitaux de Paris (PHRC AOM06053, P060245) and the Agence Nationale pour la Recherche (ANR-05-MRAR-022-01). 


\begin{abstract}
Cilia are evolutionarily conserved structures that play key roles in diverse cell types. Motile cilia are involved in the most prominent ciliopathy called primary ciliary dyskinesia (PCD) that combines respiratory symptoms, male infertility, and, in half cases, situs inversus. The diagnosis of PCD relies on the identification of ciliary abnormalities that mainly concern outer and/or inner dynein arms (ODA, IDA). PCD is a genetic condition, usually inherited as an autosomal recessive trait. To date, six genes have been clearly implicated in PCD. Two "major" genes, DNAII and DNAH5, underlie PCD in nearly half of the patients with ODA defects, whereas $R P G R, D N A H 11$ and $T X N D C 3$ are implicated in rare families with specific phenotypes (retinitis pigmentosa, abnormal beating of structurally normal cilia, and situs ambiguous, respectively). The relative contribution of DNAI2, is currently assessed. In all the other patients with ODA or other ultrastructural defects, the causative genes remain to be identify.
\end{abstract}

Key words : primary ciliary dyskinesia (PCD), cilia, genetics, Kartagener syndrome, dynein, situs inversus, mutations 


\section{Introduction}

The history of primary ciliary dyskinesia (PCD) began with the $20^{\text {th }}$ century when A. Siewert noticed the surprising association of bronchiectasis and situs inversus ${ }^{1}$. In 1933, this association was formalised by M. Kartagener who described the triad of sinusitis, bronchiectasis, and situs inversus. Some forty years later, B. Afzelius identified in the axoneme of respiratory cilia and sperm flagella of patients with Kartagener syndrome the underlying ultrastructural defect corresponding to an absence of dynein arms ${ }^{2}$. By the end of the $20^{\text {th }}$ century, DNAIl, the human ortholog of the Chlamydomonas reinhardtii gene $I C 78$, was identified as the first gene involved in $\mathrm{PCD}^{3}$, thereby opening up a new field of research to decipher the pathophysiology of this complex disorder.

\section{Ciliary structure}

Cilia are evolutionarily conserved complex structures that protrude from the apical surface of most eukaryotic cells ${ }^{4}$. These organelles, which are structurally related to the flagella of spermatozoa, can be classified according to the arrangement of their microtubule cytoskeleton core, called axoneme. The axoneme consists either of nine doublets only $(\langle 9+0\rangle$ pattern) or of nine outer-doublet microtubules surrounding a central pair of single microtubules $(« 9+2 »$ pattern). The « $9+0 »$ primary cilia are immotile, except in the embryonic node where they are involved in left-right asymmetry. In mammals, primary cilia are present on most cells where they have mechanosensory properties, acting as antennae, sensing the external environment, while sensory cilia play key roles in vision and olfaction. The $« 9+2 »$ motile cilia are always motile, are involved in the transport of extracellular fluids; for example, ciliated epithelium can be found in the respiratory tract, the brain ependyma, the female oviduct and the male vas deferent. Most importantly, respiratory cilia propel mucus along the respiratory tract and represent, therefore, the first line of airway defences ${ }^{5}$.

The axonemal proteins have been shown to be extremely conserved throughout evolution from lower eukaryotes, like the unicellular green algae Chlamydomonas, to mammals ${ }^{6}$. The axoneme of motile cilia (Fig.1) consists of $\alpha$ and $\beta$ tubulin heterodimers arranged in protofilaments to form microtubules. Each peripheral doublet is composed of an A microtubule (13 protofilaments) and a B 
microtubule (11 protofilaments) surrounding a central complex (CC) consisting of two single microtubules $(\mathrm{C} 1$ and $\mathrm{C} 2)$ inside a central sheath. Nexin links and radial spokes are multiprotein complexes that interconnect the adjacent peripheral doublets to each other and to the central microtubules, respectively. The inner dynein arms (IDA) and the outer dynein arms (ODA), bound to each A peripheral microtubules with certain periodicity, are the transducers of mechanical forces necessary for ciliary motion via a number of complex cell events including phosphorylation/dephosphorylation of key proteins ${ }^{4}$. Detailed structural and biochemical studies of dynein arms have been performed in various species including sea urchin and Chlamydomonas reinhardtii. These studies have revealed that dynein arms are large multisubunit molecular motors formed by the assembly of heavy, intermediate and light chains (HC of 400-500 kDa, IC of 45-110 $\mathrm{kDa}$ and LC of 8-55 kDa, respectively). The ODA is composed of three or two HCs $(\alpha, \beta, \gamma)$, depending on species (only $\beta$ and $\gamma$ in humans), two ICs and 8 LCs. Dynein HCs provide, through ATPase activity, the energy necessary for ciliary beating. The IDAs are more complex with at least seven different isoforms: one with two HCs and each of the six other isoforms contains a different $\mathrm{HC}$; each isoform includes different ICs and $\mathrm{LCs}^{7}$. Assembly and maintenance of cilia and flagella are dependent on intraflagellar transport (IFT). IFT is an intracellular motility system, by which large protein complexes called IFT particles are transported bidirectionally between the cell membrane and the outer doublet microtubules of cilia and flagella. IFT, which was first described in Chlamydomonas, has subsequently been found to be essential for the assembly of motile and sensory cilia in many organisms including mice ${ }^{8}$.

\section{Ciliary defects}

Cilia play key physiological and developmental roles in diverse cell types and organisms. In mammals, defects in primary cilia cause a wide range of disorders such as cystic diseases and/or sensorial disorders, while motile cilia are involved in the most prominent ciliopathy called primary ciliary dyskinesia (PCD). PCD (MIM 242650) is a rare respiratory disease due to impaired mucociliary clearance resulting from functional and ultrastructural abnormalities of respiratory cilia ${ }^{9,10}$. It represents a heterogeneous group of genetic disorders, usually beginning in early childhood. 
Approximately half of the patients with PCD display a situs inversus, thereby defining the Kartagener syndrome (MIM 244400). This complex phenotype, i.e. chronic airway infections, situs inversus and male infertility, is now clearly explained by a single event involving the axonemal structure found in motile respiratory, embryonic nodal cilia, and flagella of spermatozoa, respectively.

The diagnosis of PCD is therefore based on the identification of functional and structural abnormalities of cilia (Fig.2). In most patients with PCD, all the cilia share the same ultrastructural defect, as expected for a congenital disease. However, depending on the patients, cilia have been shown to carry various axonemal abnormalities. The first ultrastructural defect that has been reported in patients with PCD was the absence of dynein arms that are essential for ciliary motion ${ }^{11}$. The main ciliary defect concerns dynein arms in more than $80 \%$ of cases, and involves more frequently ODA than IDA ${ }^{12,13}$. Other axonemal abnormalities concern the central complex or the radial spokes (axonemal disorganization). In our 20-year experience, as in literature, the spectrum of ultrastructural defects in PCD is as follows: 24 to $43 \%$ of isolated ODA defects, 24 to $45 \%$ of defects involving both dynein arms, 14 to $29 \%$ of isolated IDA defects, and 4 to $18 \%$ of CC defects ${ }^{12-16}$. Ciliary motility seems to be related to the type of ciliary defects: most of the cilia are immotile when both dynein arms or outer dynein arms are affected ${ }^{13,14,17-19}$, while the ciliary beating pattern seems abnormal with reduced amplitude in case of inner dynein arm defects ${ }^{19}$. In case of anomalies of the central complex, the ciliary beating pattern is abnormal ${ }^{12,17,20}$. In $10-20 \%$ of patients, no ultrastructural defects can be found through conventional electron microscopy, although cilia are immotile $^{3,5,14,21,22}$. In these patients, the diagnosis of PCD is based on the presence of typical clinical features (including situs inversus) with the absence of ciliary motion. In these cases, a low level of nasal nitric oxide might be very helpful to assert the diagnosis of PCD. It has been proposed that the defect could concern some enzymes associated with various axonemal structures ${ }^{23}$. In fact, Kartagener syndrome with normal cilia is not an exceptional condition ${ }^{24-26}$. As a consequence, and because of random lateralization, it is tempting to speculate that the same proportion of "true" patients with PCD, but with normal cilia and without situs inversus, should exist. 


\section{PCD inheritance}

In most instance, the transmission pattern of PCD is consistant autosomal recessive inheritance of the disease ${ }^{11}$, although rare families showing autosomal dominant or X-linked modes of inheritance have also been reported ${ }^{27-29}$. The incidence of PCD ranges from 1/15,000 to $1 / 60,000$ alive births ${ }^{11}$, while that of Kartagener syndrome is estimated to range between $1 / 30,000$ and $1 / 20,000$ live births. However, the frequency of the disease is markedly underestimated, especially when situs inversus is absent and/or when ultrastructure is normal.

As predicted from the high complexity of ciliary structure and function, there is ample room for genetic heterogeneity in PCD. Each of the several hundreds of proteins that constitute a cilia could potentially cause the disease. Such complexity makes approaches based on genetic linkage difficult to apply; except in case of large and/or consanguineous families, a situation which is exceptional. Alternative approaches rely on the fact that (i) the structure of the ciliary axoneme is highly conserved across species and (ii) the protein components of a protozoan axoneme, such as those found in Chlamydomonas reinhardtii, and those of mammalian cilia are highly homologous at the sequence level ${ }^{6}$. Strikingly, several immotile strains of Chlamydomonas carry axonemal ultrastructural defects that are reminiscent of those reported in patients with PCD; this observation led to the development of a candidate-gene approach based on Chlamydomonas mutants. It enabled the characterization of the first gene involved in PCD, called DNAII that encodes a dynein intermediate chain of the $\mathrm{ODA}^{3}$.

\section{PCD genes (Table I)}

DNAI1, which is located in the p21-p23 region of chromosome 9 , comprises 20 exons spanning about $62 \mathrm{~kb}$ of genomic DNA. It encodes a 699 -amino-acid protein orthologous to the Chlamydomonas ODA protein IC78. Mutations in DNAIl have been found in several PCD patients including patients with Kartagener syndrome ${ }^{3,30-32}$. Although allelic heterogeneity was noted in the very first studies, it was secondarily shown that the IVS1+2_3insT mutation, which accounts for about half the mutated alleles, results in fact from a founder effect ${ }^{32}$. As so far, mutations in DNAII 
have always been found in association with ODA defects and account for about $14 \%$ of those patients $^{32}$.

Thanks to a genetic linkage analysis performed in one large consanguineous multiplex family, mutations have been identified in a second gene encoding a dynein heavy chain, $\operatorname{DNAH}^{33} 34$. DNAH5, located on chromosome 5, codes for a protein orthologous of the Chlamydomonas $\gamma$ axonemal heavy chain of the ODA. The human DNAH5 gene, which spans $250 \mathrm{~kb}$, comprises 80 exons. So far, DNAH5 mutations have been found only in patients with partial or total loss of ODA associated or not with left-right asymmetry anomalies ${ }^{34,35}$. The DNAH5 mutation detection rate was shown to reach $49 \%$ in a subset of PCD families with documented ODA defects ${ }^{35}$.

A third candidate gene (DNAH11) has first been involved in a patient with a complex phenotype associating cystic fibrosis, situs inversus and a paternal uniparental isodisomy of chromosome 7, a chromosome that carries both the CFTR and DNAH11 genes ${ }^{36}$. DNAH11, which encodes also a dynein heavy chain, comprises 82 exons, spanning more than $353 \mathrm{~kb}$ of the $\mathrm{p} 21$ region of chromosome 7. The patient was shown to be homozygous for the F508del mutation of the CFTR gene, and for the R2852X nonsense mutation in DNAH11. Notably, a missense mutation had been identified in the Dnahll gene of the iv/iv (inversus viscerum) mouse characterized by immotile cilia of the embryonic node and random organ lateralization ${ }^{37}$. Secondarily, compound heterozygous DNAH11 mutations were identified in a large family in which five individuals have PCD and one has Kartagener syndrome. Respiratory cilia from affected individuals showed a normal axonemal ultrastructure but an abnormal ciliary beating pattern with a reduced bending capacity and a hyperkinetic beat. Mutations in DNAHI1, therefore, do not result in axonemal ultrastructural defect detectable by electron microscopy, but are believed to affect motor function ${ }^{26}$.

PCD may also be caused by mutations in RPGR (retinitis pigmentosa guanosine triphosphatase regulator), the most common gene involved in X-linked retinitis pigmentosa or TXNDC3 that codes for a protein belonging to the thioredoxin superfamily. The $R P G R$ gene, located on the $\mathrm{p} 21.1$ region of the chromosome $\mathrm{X}$ is essential for photoreceptor maintenance and viability; it is also expressed in respiratory ciliated cells ${ }^{38}$. In rare instances, typical symptoms of PCD have been reported in males 
with retinitis pigmentosa ${ }^{28,39,40}$. More recently, a 57-bp intragenic deletion of this gene has been identified in one family in which two boys present a complex phenotype combining PCD with retinitis pigmentosa. Ciliary investigations showed immotile cilia and various ultrastructural abnormalities with partial dynein arm defects ${ }^{29}$. This finding, which underlines the importance of $R P G R$ in the development of axonemal structures in photoreceptors and in respiratory cilia, represents the first clear demonstration of an X-linked transmission of the PCD phenotype. TXNDC3, which encodes a thioredoxine-nucleoside diphosphate kinase, is expressed in testis and respiratory epithelial cells. This gene was considered as a candidate for PCD because it represents the human ortholog of the sea urchin $I C l$ gene that encodes a component of sperm $\mathrm{ODA}^{41}$; it was therefore tested in a series patients with PCD and ODA abnormalities. In a young girl with PCD and situs ambiguous was identified a nonsense mutation combined, in trans, with an intronic SNP controlling the respective amounts of two physiological $T X N D C 3$ isoforms generated by alternative splicing ${ }^{42}$ and shown to display different binding properties to microtubules.

Just recently, several loss-of-function mutations have been identified in the DNAI2 gene of patients with ODA defect ${ }^{43}$, therefore confirming that DNAI2 which is located on the $\mathrm{q} 25$ region of chromosome 17, was indeed a good candidate gene for PCD ${ }^{44}$.

A number of other candidate genes for PCD (such as DNAH9, HP28, DPCD, SPAG16, HFH-4) ${ }^{45}$ have been selected for mutation screening in PCD patients, but, so far, no mutations have been reported. In addition, approaches based on positional cloning have pointed to several possible PCD loci (e.g. 1q23.1-q32.1, 7p14.2-12.2, 15q13.1-15.1, 15q24-25, 16p12.1-12.2, 19q13.3-qter regions) ${ }^{46-}$ 51; the causative genes are still to be identified.

\section{Conclusion}

The molecular basis of PCD is just beginning to be elucidated. To date, despite substantial efforts made for many years by several groups and the recent availability of huge sequence information, only six genes (DNAI1, DNAH5, DNAH11, RPGR, TXNDC3 and DNAI2) have been clearly implicated in PCD. Two of them, DNAII and DNAH5, which underly the disease in about $25 \%$ of the patients with 
PCD, represent major genes, while three others (RPGR, DNAH1 l, TXNDC3), are "minor" genes, implicated in only few families. The relative contribution of DNAI2 to the pathology remains to be determined. Although DNAIl and DNAH5 mutations underlie PCD in nearly half of patients with ODA defects, for all the other patients with ODA defects or with other ultrastructural phenotypes, the genes responsible for their ciliary defect are still to be identified. This is an important task for a better management of the disease (e.g. development of new diagnostic methods and of more efficient treatments). This research should also provide new insights into the molecular mechanisms involved in the assembly and function of cilia, as well as in the determination of laterality. 


\section{References}

1. Siewert. Ueber einen fall von bronchiectasie bei einem patienten mit situs inversus viscerum. Berliner klinische wochenschrift 1904; 41: 139-41.

2. Kartagener, M. Zur pathogenese der bronchiektasien. Bronchiektasien bie situs inversus viscerum. Beitr Klin Tuberk 1933; 83: 489-501.

3. Pennarun, G, Escudier, E, Chapelin, C et al. Loss-of-function mutations in a human gene related to Chlamydomonas reinhardtii dynein IC78 result in primary ciliary dyskinesia. Am J Hum Genet 1999; 65: 150819.

4. Haimo, L TRosenbaum, J L. Cilia, flagella, and microtubules. J Cell Biol 1981; 1991: 125s-30s.

5. Satir, PChristensen, S T. Overview of structure and function of mammalian cilia. Annu Rev Physiol 2007; 17: $377-400$.

6. Hook, PVallee, R B. The dynein family at a glance. J Cell Sci 2006; 119: 4369-71.

7. Porter, M E. Axonemal dyneins: assembly, organization, and regulation. Curr Opin Cell Biol 1996; 8: 10-7.

8. Rosenbaum, J LWitman, G B. Intraflagellar transport. Nat Rev Mol Cell Biol 2002; 3: 813-25.

9. Afzelius, B A. A human syndrome caused by immotile cilia. Science 1976; 193: 317-9.

10. Rossman, C M, Forrest, J B, Ruffin, R ENewhouse, M T. Immotile cilia syndrome in persons with and without Kartagener's syndrome. Am Rev Respir Dis 1980; 121: 1011-6.

11. Afzelius, B A. The immotile-cilia syndrome: a microtubule-associated defect. CRC Crit Rev Biochem 1985; 19: 63-87. 
12. Chilvers, M A, Rutman, AO'Callaghan, C. Ciliary beat pattern is associated with specific ultrastructural defects in primary ciliary dyskinesia. J Allergy Clin Immunol 2003; 112: 518-24.

13. Noone, P G, Leigh, M W, Sannuti, A et al. Primary ciliary dyskinesia: diagnostic and phenotypic features. Am J Respir Crit Care Med 2004; 169: 459-67.

14. De Iongh, R URutland, J. Ciliary defects in healthy subjects, bronchiectasis, and primary ciliary dyskinesia. Am J Respir Crit Care Med 1995; 151: 1559-67.

15. Jorissen, M, Willems, T, Van der Schueren, B, Verbeken, EDe Boeck, K. Ultrastructural expression of primary ciliary dyskinesia after ciliogenesis in culture. Acta Otorhinolaryngol Belg 2000; 54: 343-56.

16. Tamalet, A, Clement, A, Roudot-Thoraval, $\mathrm{F}$ et al. Abnormal central complex is a marker of severity in the presence of partial ciliary defect. Pediatrics 2001; 108: E86.

17. Rossman, C M, Forrest, J B, Lee, R M, Newhouse, A FNewhouse, M T. The dyskinetic cilia syndrome; abnormal ciliary motility in association with abnormal ciliary ultrastructure. Chest 1981; 80: 860-5.

18. Pedersen, MMygind, N. Rhinitis, sinusitis and otitis media in Kartagener's syndrome (primary ciliary dyskinesia). Clin Otolaryngol 1982; 7: 373-80.

19. Chilvers, M A, Rutman, AO'Callaghan, C. Functional analysis of cilia and ciliated epithelial ultrastructure in healthy children and young adults. Thorax 2003; 58: 333-8.

20. Escalier, D, Jouannet, PDavid, G. Abnormalities of the ciliary axonemal complex in children: an ultrastructural cinetic study in a series of 34 cases. Biol Cell 1982; 44: 271-82.

21. Chao, J, Turner, J ASturgess, J M. Genetic heterogeneity of dynein-deficiency in cilia from patients with respiratory disease. Am Rev Respir Dis 1982; 126: 302-5. 
22. Fliegauf, M, Olbrich, H, Horvath, J et al. Mislocalization of DNAH5 and DNAH9 in respiratory cells from patients with primary ciliary dyskinesia. Am J Respir Crit Care Med 2005; 171: 1343-9.

23. Porter, M ESale, W S. The $9+2$ axoneme anchors multiple inner arm dyneins and a network of kinases and phosphatases that control motility. J Cell Biol 2000; 151: F37-42.

24. Greenstone, M, Rutman, A, Pavia, D, Lawrence, DCole, P J. Normal axonemal structure and function in Kartagener's syndrome: an explicable paradox. Thorax 1985; 40: 956-7.

25. Escudier, E, Escalier, D, Homasson, J P, Pinchon, M CBernaudin, J F. Unexpectedly normal cilia and spermatozoa in an infertile man with Kartagener's syndrome. Eur J Respir Dis 1987; 70: 180-6.

26. Schwabe, G C, Hoffmann, K, Loges, N T et al. Primary ciliary dyskinesia associated with normal axoneme ultrastructure is caused by DNAH11 mutations. Hum Mutat 2008; 29: 289-298.

27. Narayan, D, Krishnan, S N, Upender, M et al. Unusual inheritance of primary ciliary dyskinesia (Kartagener's syndrome). J Med Genet 1994; 31: 493-6.

28. Krawczynski, M RWitt, M. PCD and RP: X-linked inheritance of both disorders? Pediatr Pulmonol 2004; 38: $88-9$

29. Moore, A, Escudier, E, Roger, G et al. RPGR is mutated in patients with a complex X linked phenotype combining primary ciliary dyskinesia and retinitis pigmentosa. J Med Genet 2006; 43: 326-333.

30. Guichard, C, Harricane, M, Lafitte, J et al. Axonemal dynein intermediate-chain gene (DNAI1) mutations result in situs inversus and primary ciliary dyskinesia (Kartagener syndrome). Am J Hum Genet 2001; 68: 10301035. 
31. Zariwala, M, Noone, P, Sannuti, A et al. Germline mutations in an intermediate chain dynein cause primary ciliary dyskinesia. Am J Respir Cell Mol Biol 2001; 25: 577-583.

32. Zariwala, M A, Leigh, M W, Ceppa, F et al. Mutations of DNAI1 in primary ciliary dyskinesia: evidence of founder effect in a common mutation. Am J Respir Crit Care Med 2006; 174: 858-66.

33. Omran, H, Haffner, K, Volkel, A et al. Homozygosity mapping of a gene locus for primary ciliary dyskinesia on chromosome 5p and identification of the heavy dynein chain DNAH5 as a candidate gene. Am J Respir Cell Mol Biol 2000; 23: 696-702.

34. Olbrich, H, Haffner, K, Kispert, A et al. Mutations in DNAH5 cause primary ciliary dyskinesia and randomization of left-right asymmetry. Nat Genet 2002; 30: 143-4.

35. Hornef, N, Olbrich, H, Horvath, J et al. DNAH5 mutations are a common cause of primary ciliary dyskinesia with outer dynein arm defects. Am J Resp Crit Care Med 2006; 174: 120-6.

36. Bartoloni, L, Blouin, J L, Pan, Y et al. Mutations in the DNAH11 (axonemal heavy chain dynein type 11) gene cause one form of situs inversus totalis and most likely primary ciliary dyskinesia. Proc Natl Acad Sci U S A 2002; 99: 10282-6.

37. Supp, D M, Witte, D P, Potter, S SBrueckner, M. Mutation of an axonemal dynein affects left-right asymmetry in inversus viscerum mice. Nature 1997; 389: 963-6.

38. Hong, D H, Pawlyk, B, Sokolov, M et al. RPGR isoforms in photoreceptor connecting cilia and the transitional zone of motile cilia. Invest Ophtalmol Vis Sci 2003; 44: 2413-21.

39. Iannaccone, A, Breuer, D K, Wang, X F et al. Clinical and immunohistochemical evidence for an X linked retinitis pigmentosa syndrome with recurrent infections and hearing loss in association with an RPGR mutation 2003; 40: 509-15. 
40. Zito, I, Downes, S M, Patel, R J et al. RPGR mutation associated with retinitis pigmentosa, impaired hearing, and sinorespiratory infections. J Med Genet 2003; 40: 609-615.

41. Ogawa, K, Takai, H, Ogiwara, A et al. Is outer arm dynein intermediate chain 1 multifunctional? Mol Biol Cell 1996; 7: 1895-907

42. Duriez, B, Duquesnoy, P, Escudier, E et al. A common variant in combination with a nonsense mutation in a member of the thioredoxin family causes primary ciliary dyskinesia. Proc Natl Acad Sci USA 2007; 104: $3336-$ 3341.

43. Loges, N T, Olbrich, H, Fenske, L et al. DNAI2 mutations cause primary ciliary dyskinesia with defects in the outer dynein arm. Am J Hum Genet (Epub ahead of print) 2008;

44. Pennarun, G, Chapelin, C, Escudier, E et al. The human dynein intermediate chain 2 gene (DNAI2): cloning, mapping, expression pattern, and evaluation as a candidate for primary ciliary dyskinesia. Hum Genet 2000; 107 : 642-9.

45. Zariwala, M, Knowles, MOmran, H. Genetic defects in ciliary structure and function. Annu Rev Physiol $2007 ; 69: 423-50$.

46. Blouin, J, Meeks, M, Radhakrishna, U et al. Primary ciliary dyskinesia: a genome-wide linkage analysis reveals extensive locus heterogeneity. Eur J Hum Genet 2000; 8: 109-118.

47. Meeks, M, Walne, A, Spiden, S et al. A locus for primary ciliary dyskinesia maps to chromosome 19q. J Med Genet 2000; 37: 241-4.

48. Jeganathan, D, Chodhari, R, Meeks, M et al. Loci for primary ciliary dyskinesia map to chromosome 16p12.1-12.2 and 15q13.1-15.1 in Faroe Islands and Israeli Druze genetic isolates. J Med Genet 2004; 41: 233 40. 
49. Geremek, M, Zietkiewicz, E, Dieh1, S R, Alizadeh, B Z, Wijmenga, CWitt, M. Linkage analysis localises a Kartagener syndrome gene to a $3.5 \mathrm{cM}$ region on chromosome 15q24-25. J Med Genet 2006; 43: e1.

50. Gutierrez-Roelens, I, Sluysmans, T, Jorissen, M, Amyere, MVikkula, M. Localization of candidate regions for a novel gene for Kartagener syndrome. Eur J Hum Genet 2006; 14: 809-15.

51. Geremek, M, Schoenmaker, F, Zietkiewicz, E et al. Sequence analysis of 21 genes located in the Kartagener syndrome linkage region on chromosome 15q. Eur J Hum Genet 2008; 16: 688-95. 
Table I: Clinical and ultrastructural phenotype according to the genes involved in PCD

\begin{tabular}{cccc}
\hline Genes & $\begin{array}{c}\text { Extra-respiratory } \\
\text { phenotypic features }\end{array}$ & Ultrastructural defect & References \\
\hline DNAI1 & \pm situs inversus & lack of ODA & $3,30-32$ \\
DNAH5 & \pm situs inversus & lack of ODA & 34,35 \\
DNAH11 & \pm situs inversus & normal axoneme & 36,26 \\
RPGR & retinitis pigmentosa & complex dynein arm defect & 29 \\
TXNDC3 & situs ambiguous & partial lack of ODA & 42 \\
DNAI2 & \pm situs inversus & lack of ODA & 43 \\
\hline
\end{tabular}


Figure 1: Schematic representation of a transversal section of the axoneme of motile cilia

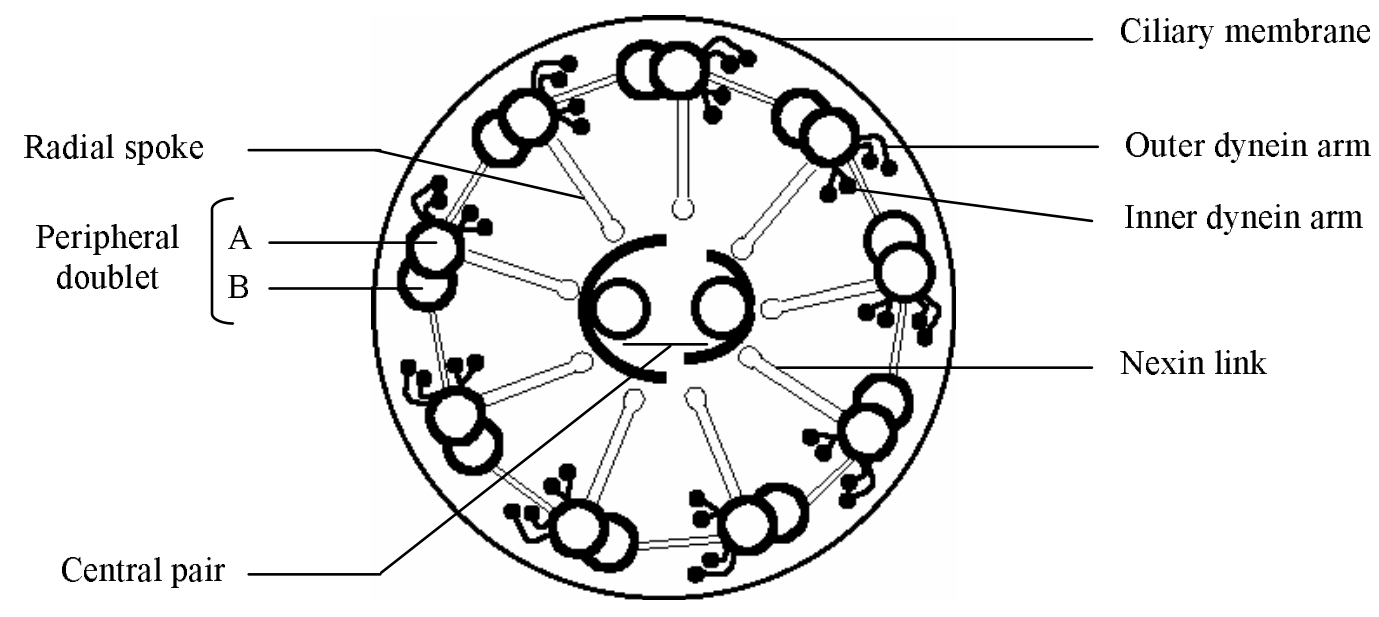


Figure 2: Normal axonemal ultrastructure of respiratory cilia and main defects found in patients with PCD
A. Cross section of a normal cilium showing the " $9+2 "$ microtubule doublet configuration with presence of both dynein arms, as shown by TEM (bar $=0.1 \mu \mathrm{m})$
B. Absence of outer dynein arms
C. Absence of both dynein arms
D. Absence of inner dynein arms combined with axonemal disorganization
E. Absence of central complex
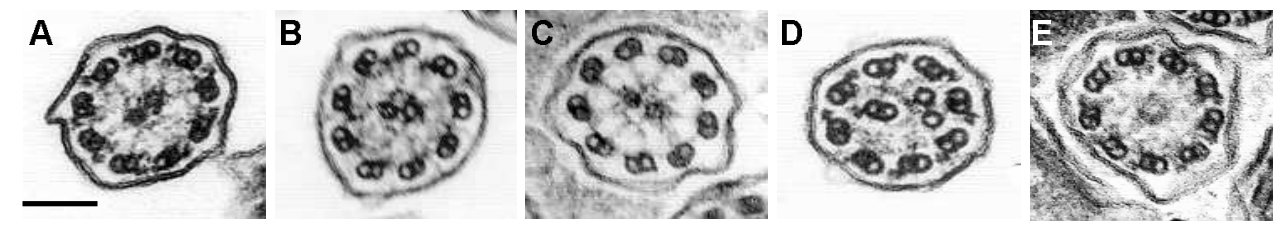